\title{
A CASE OF CRATER-LIKE HOLE IN THE OPTIC DISC
}

BY

\section{J. H. DOGgaRT}

LONDON

A MARRIED woman, aged 31 years, recently attended Mr. R. R. James' Out-patients at St. George's Hospital, complaining of headaches. Vision of $6 / 9$ and $6 / 6$ is obtained in the right and left eyes respectively after correction of a small amount of compound myopic astigmatism. The left fundus is normal. In the right, there is a large inferior crescent showing heaped-up pigment along most of its lower border. An oval, greyish-blue hole is situated in the infero-temporal quadrant of the optic disc, lying with its long axis in the direction of the long axis of the disc. The depth of this hole is about $9 \mathrm{D}$. No vessel is visible on the floor, although two smaller ones can be seen in close relation to its margins. The macular region is normal.

Since the original description of this rare congenital anomaly by Wiethe in 1882, about forty such cases, two-thirds of them in women, have been recorded. The holes are usually unilateral,

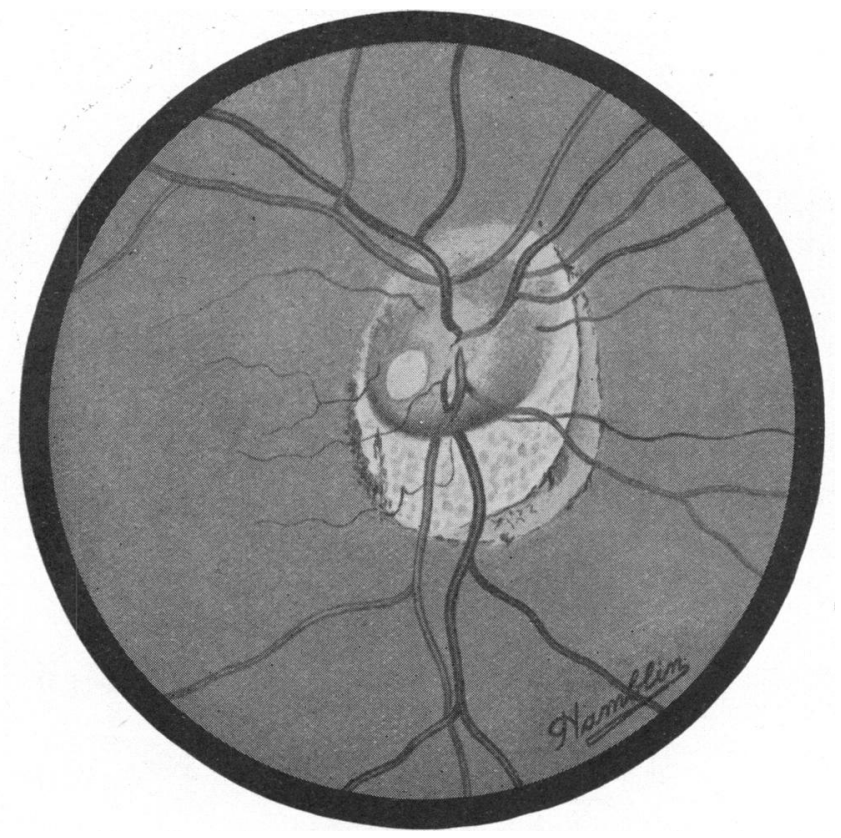

FIG. 1. 
single, not associated with defective vision, and appear by ophthalmoscopic estimation to be about $10 \mathrm{D}$ in depth. They are nearly always situated in the infero-temporal quadrant of the disc. Bloodvessels are seldom seen on the floor. In a few cases, the clinical picture has been complicated by pigmentary changes at the macula, with corresponding defects in the central field. Occasionally, as in the present instance, there is a crescentic or irregular extension of the disc in the neighbourhood of the hole. There are also several examples of a disc with more than one hole. It is matter for curiosity that, in van de Hoeve's patient with three holes in the right disc, the only unaffected quadrant was the infero-temporal. Paracentral scotoma attributable to crater-like hole is said to occur. In the case I record here, it is impossible to blame the hole for any of the irregular field defects mapped out on the accompanying charts. These defects might well be due entirely to the inferior crescent. In the central portion of the right field, plotted out on a

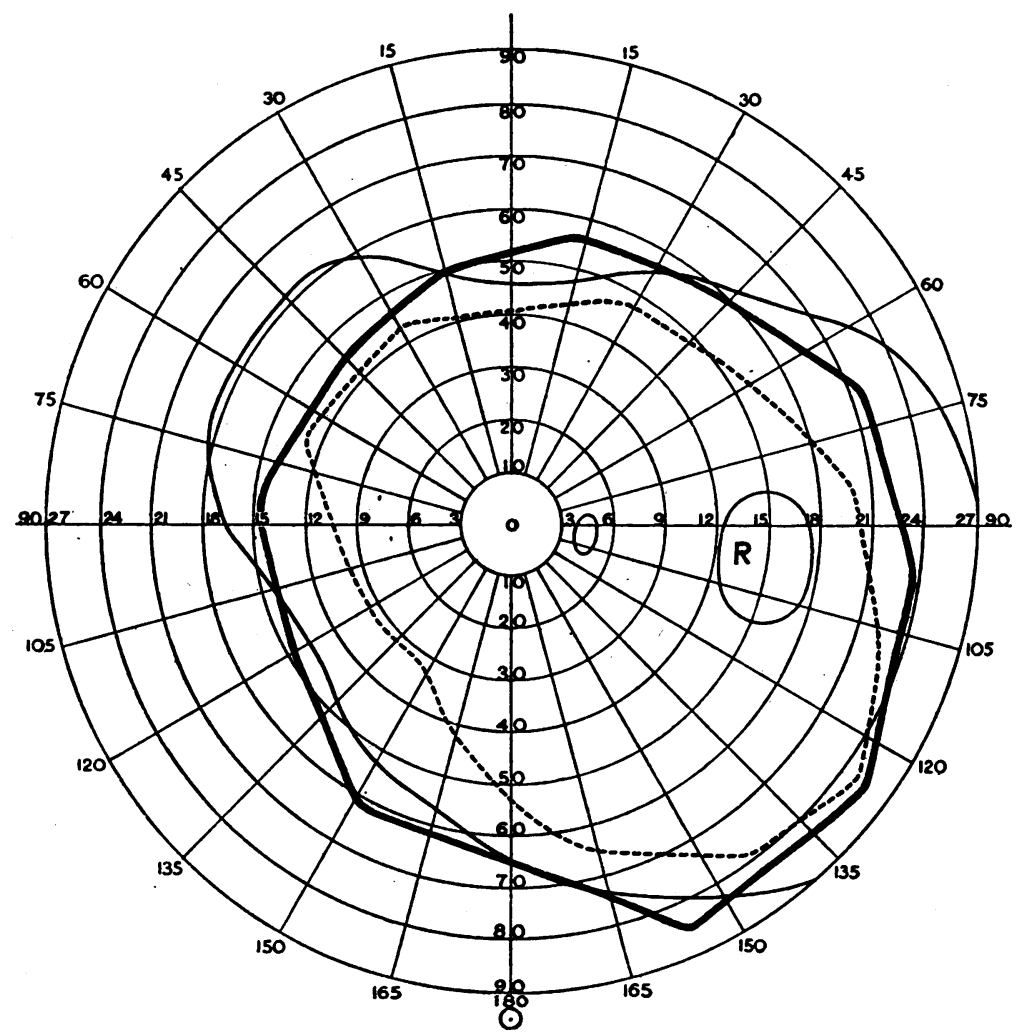

FIG. 2.

Thick black line $=$ field plotted with $10 / 330$ moving white object . Dotted line $=1 / 330$ object. 


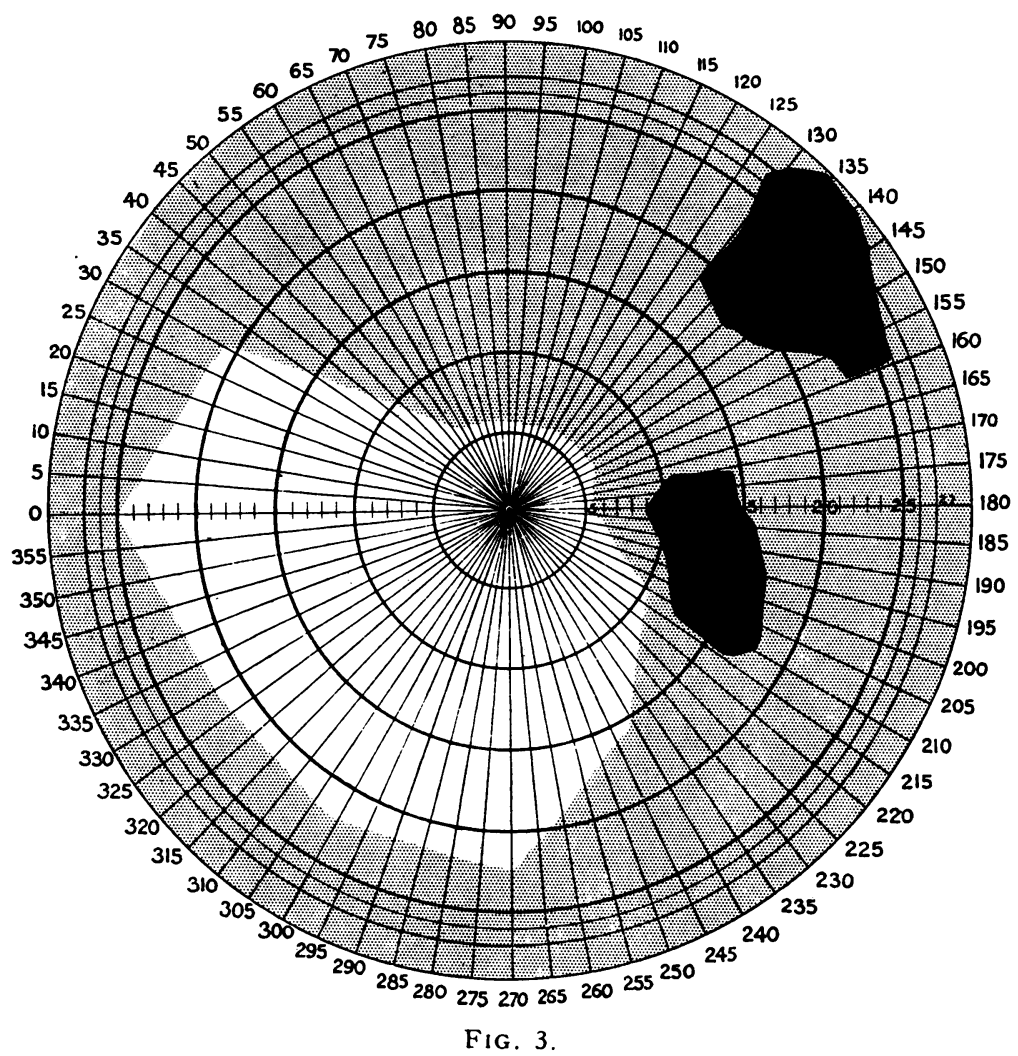

Black area $=$ enlarged blind spot, plotted with $10 / 2,000$ object. Dark grey area $=$ deficiency for $4 / 2,000$ object. Light grey area=deficiency for $2 / 2,000$ object.

Bjerrum screen by Traquair's method, there is an enlargement of the blind spot; a large relative scotoma supero-temporally; and a wide area of relative defect for the smallest object used. Control observation on the unaffected eye reveals no abnormality. The central field of this patient's right eye affords an illustration of the extensive defects compatible with good test-type vision and a fairly good peripheral field. Indeed, the actual discrepancy between the two eyes is even greater than the chart would indicate, because the smallest feasible test-object for the right eye is $2 \mathrm{~mm}$. in diameter. The $1 \mathrm{~mm}$. object, whose presence the left eye can appreciate over almost the entire screen, is invisible to the right eye, save in the region of the fixation-point.

To Mr. James, who himself published a case of this anomaly in 1913, I wish to express my thanks for allowing me to publish this report. A bibliography was given in The British Journal of Ophthalmology, Vol. XI, p. 16, 1927. 